\title{
Ein Blick hinter das Schweizer "Gedächtnistheater": Jüdische Jugendliche in Zürich
}

\author{
*itta.baver@geo.uzh.ch, Geographisches Institut der Universität Zürich \\ mit Beiträgen von Kerstin Fankhauser, Tanja Frei, Seraina Klaus, Philipp Rüdlisühli und Raphael Zurfluh
}

eingereicht am: 20.12.2019, akzeptiert am: 05.02.2020

\begin{abstract}
Aufbauend auf den provokativen Thesen von Max Czollek möchte der Beitrag danach fragen, wie es um das „Gedächtnistheater" in der Schweiz bestellt ist. Wie positionieren sich jüdische Jugendliche in Zürich? Welchen Stellenwert nehmen Formen von Aktivismus von Jugendlichen und für Jugendliche ein? Mit diesen Fragen beschäftigten sich angehende Geographielehrer/innen (Gymnasien) im Rahmen eines Forschungs- und Lehrmoduls. In diesem Zusammenhang nahmen die Studierenden auch an einem Schulbesuch von zwei jüdischen Jugendlichen teil, die sich für das Projekt „Likrat“ engagieren. Dieses vom Schweizer Israelitischen Gemeindebund (SIG) geförderte Projekt schult junge Jüdinnen und Juden, damit sie jeweils zu zweit Schulbesuche in der Schweiz durchführen können. In offenen Gesprächskreisen werden unter Jugendlichen heikle Themen, wie etwa Antisemitismus, Shoa oder jüdische Kultur in der Schweiz diskutiert. Damit wird ein wichtiger Beitrag zur Prävention von Rassismus und Antisemitismus geleistet.
\end{abstract}

Keywords: Geographie und Geschichte, Erinnerungskultur, Schweiz, jüdische Jugendliche

\section{Looking behind Swiss "Gedächtnistheater" (= remembrance theatre): Jewish adolescents in Zurich}

This paper starts from the provocative arguments put forward by Max Czollek in his book „Desintegriert euch!“ (2018) and elaborates on how young people in Switzerland negotiate what Czollek calls "Gedächtnistheater". For this purpose, a group of students worked on different perspectives of young Jews living in Zurich, Switzerland. The ideas discussed in this seminar were put into practice when we participated in a school visit of Likrat. Likrat is a program that educates, organizes and supports young people with a Jewish background who, in pairs, visit school classes and offer an open exchange between Jewish and non-Jewish young people. In an interview with two young people from Likrat, we discussed the thesis elaborated during the seminar and learnt what it is like to live as a young Jew in Zurich.

Keywords: Geography and history, remembrance Switzerland, Jewish youth

\section{$1 \quad$ Hintergrund und Ziel des Beitrags}

Das „Gedächtnistheater“, um das es in diesem Artikel gehen soll, stellt sich am besten selbst vor. Treffenderweise auch gleich in geeigneter Form: szenisch und performativ.

[Deutscher Journalist interviewt jüdische Künstlerin:] Was ist die Geschichte ihrer Familie? Also, ich meine im Zweiten Weltkrieg, also den Holocaust, also haben Sie Familie im Holocaust verloren? Und was ist Ihre Erfahrung mit Antisemitismus? Also ich meine heute in Deutschland, haben Sie schon mal Antisemitismus erlebt? Und noch was: Haben Sie eigentlich Verwandte in Israel? Ich war ja auch mal da. Das Essen, wunderbar, und die Leute sind so hübsch. Besonders die Frauen, aber auch die Männer. Jaja, das macht die Armee aus Menschen. Nur schade das mit den Palästinensern, könnte doch so ein tolles Land sein! (Czollek 2018a: 84)

Diese und ähnliche Szenen deutsch-jüdischer Begegnungen bezeichnet der Soziologe Michal Bodemann als „Gedächtnistheater“, weil sie einem „kreativen und dramatischen Akt" auf der Bühne gleichen (Bodemann 1996: 183). Drei Elemente sind konstituierende Bestandteile dieses Schauspiels: Erstens, „deutsche 
Täter/innen und ihre Nachkommen treffen auf jüdische Opfer und inszenieren ihre Läuterung". Zweitens erfüllt es die Funktion „kollektiver Identitätsstiftung“, indem es dazu dient ein neues deutsches Selbstbild $\mathrm{zu}$ konstruieren. Und drittens ist es ein Ausdruck von Trauer über eine Gewalttat (Czollek 2018a: 84). Insbesondere die ersten beiden Elemente stehen im Mittelpunkt von Max Czolleks Kampfschrift „Desintegriert euch!“, die das deutsche Selbstverständnis im Jahr 2018 kräftig durchgeschüttelt hat (Czollek 2018b). Der Berliner Lyriker und Essayist provoziert in seinem Buch (2018b) eine breite Öffentlichkeit mit seinen Thesen, die sich pointiert zusammenfassen lassen: Die Deutschen (miss) brauchen Jüdinnen und Juden für ihre eigene Vergangenheitsbewältigung! Dabei ist diese Provokation im Grunde ein medial geschickt inszenierter Remix von Gedanken, die Michal Y. Bodemann zum "Gedächtnistheater" bereits 1996 in einer historisch-soziologischen Analyse vorgelegt hat. Darin heißt es u.a.: „Die Darstellung der Juden als still leidende Opfer ist ein besonderes Charakteristikum des deutschen Gedächtnistheaters, das von seinen jüdischen Mitspielern weitgehend akzeptiert wird. (...) Doch eben an dem Punkt, an dem das Gedenken in nationale Gedenkfeiern umgemünzt wird, werden Juden gebraucht - die toten Juden und die lebendigen Körper von Juden“ (Bodemann 1996: 115).

Bodemann und Czollek vertreten den Standpunkt, dass das deutsche „Gedächtnistheater" auf der Pointe der Versöhnung basiert, für die Jüdinnen und Juden eine wichtige Nebenrolle spielen. Sie werden als passive Statistinnen und Statisten wahlweise auf historisch oder politisch gebohnerte Bühnen der deutschen Selbstinszenierung gezerrt. Dabei bleiben die Stimmen von deutschen Jüdinnen und Juden selbst ungehört-stumm oder berechenbar-affirmativ. Im gleichen Buch verleiht Max Czollek (2018b) vehement auch seiner Forderung „Desintegriert euch!“ Ausdruck: „Desintegration ist (...) eine Erwiderung auf die beständig vorgetragene politische und gesellschaftliche Forderung nach Integration. (...) Wenn ich vom Integrationsdenken oder vom Integrationsparadigma schreibe, dann meine ich die Konstruktion eines kulturellen und politischen Zentrums, das sich implizit oder ausdrücklich als deutsch versteht. Ich behaupte, dass das Denken in Kategorien der Integration und Leitkultur die Phantasien von ethnischer Homogenität und kultureller Dominanz nicht nur nicht verhindern kann, sondern seinen Anteil daran hat, dass diese Konzepte nicht auf dem Schrottplatz der Geschichte bleiben, auf den sie gehören. Mit dem Konzept der Desintegration schlage ich ein Gesellschaftsmodell vor, das solche neovölkischen Vorstellungen unmöglich macht." (2018b: 15; Herv. i. O.) Die Forderung „Desintegriert euch!“ ist aber nicht nur an die Adresse von jüdischen Mitbürgerinnen und Mitbürgern gerichtet, die sich nach Czolleks Ansicht präsenter und selbstbewusster für die eigene Geschichtsschreibung und die eigene gesellschaftliche Position in der Mehrheitsgesellschaft einsetzen sollten. Es ist vielmehr ein gesamtgesellschaftlich angelegter Aufruf, der auf eine konkrete Utopie einer Gesellschaft der radikalen Diversität abzielt, in dem die Stimmen von verschiedensozial-kodierten Minderheiten nicht länger überhört werden, weil sie selbstbewusster, radikaler und provozierender auftreten und sich so einen Resonanzraum in der Echtzeit der Mehrheitsgesellschaft erkämpfen.

Im vorliegenden Artikel wird es darum gehen, diesen beiden eng miteinander verwobenen Argumentationssträngen nachzugehen. Empirische Beispiele aus einem Modul mit und für Lehrdiplom-Studierende der Eidgenössisch-Technischen Universität Zürich (ETHZ) zeigen auf, wie die oben angestellten Überlegungen zum Gedächtnistheater und zur Desintegrationsthese in die Hochschullehre aufgenommen werden können. Dabei ist es wichtig herauszustellen, dass hier auch ein Wechsel des nationalen Kontextes von Deutschland zur Schweiz gedanklich (nach)vollzogen werden muss.

\section{Werkstattbericht aus einem Seminar für Lehrdiplom-Studierende}

Im Frühjahrssemester 2019 bot ich für fünf Lehrdiplom-Studierende (Geographie) das Modul „Lernorte für Geographie und Geographiedidaktik" an. Als thematisches Rahmenthema wählte ich „Jüdinnen und Juden in der Schweiz", was nicht nur eine inhaltliche, sondern auch eine didaktische Herausforderung darstellte. Während in Deutschland bereits ansatzweise aufgearbeitet wurde, welche Position und Rolle die Geographie als Wissenschaft (Michel 2014; Rössler 1986; Schultz 1989) und das Schulfach Geographie oder Erdkunde (Heske 2008; Schultz 2017) im Nationalsozialismus spielten, so wurde die z.T. faschistische Vergangenheit der Geographie in der Schweiz bis heute nur ansatzweise thematisiert (Siegrist 1989). ${ }^{1}$

Ein Vorwissen in Bezug auf die Disziplinentwicklung oder verschiedene Wissenschaftstraditionen und -ansätze in der Humangeographie und Geographiedidaktik kann bei der von mir unterrichteten Gruppe Studierender nicht vorausgesetzt werden. Die Seminarteilnehmer/innen verfügten zwar über eine fundierte naturwissenschaftliche Ausbildung auf der Bachelor- und Masterstufe, Module in Humangeographie

Dieser Frage wird aktuell in einem Modul zu „Geographie und Aktivismus“ am Geographischen Institut der Universität Zürich nachgegangen. Weitere Informationen unter: https://www.geo. uzh.ch/de/department/125/blog/geographie-in-aufruhr.html, 27.01.2020 
und Geographiedidaktik stellen dagegen in ihrem Studium nur einen sehr geringen Anteil dar. Dies wäre zwar eine wünschenswerte Grundlage für das Modul, ist allerdings auch nicht zwingend notwendig, weil es hier nicht um ontologische Grundsatzfragen geht, sondern um eine konkrete Auseinandersetzung mit den Thesen von Max Czollek, der Situation von Jüdinnen und Juden in Zürich und einem Begegnungsprojekt namens "Likrat“. Weil alle Studierende das Lehrdiplom anstreben und in ihrer späteren Berufspraxis Geographie als sehr breites Schulfach vertreten werden, wollte ich bewusst ein sozialwissenschaftliches Thema setzen, das eine Auseinandersetzung mit humangeographischen und gesellschafts-historischen Aspekten anregen sollte und einen klar erkennbaren lokalen Bezug zu Zürich herstellte.

Aus den angebotenen Themen wählten die Studierenden folgende Titel für ihre individuellen Arbeiten:

- Historische Entwicklung der jüdischen Gemeinde in Zürich im Vergleich mit Mediendarstellung von Jüdinnen/Juden in Zürich

- Synagoge als Zentrum jüdischen Glaubens

- Orte jüdischen Lebens und Wirtschaftens

- Wie leben jugendliche Juden und Jüdinnen in Zürich?

- Orte antisemitischer oder diskriminierender Vorfälle in Zürich

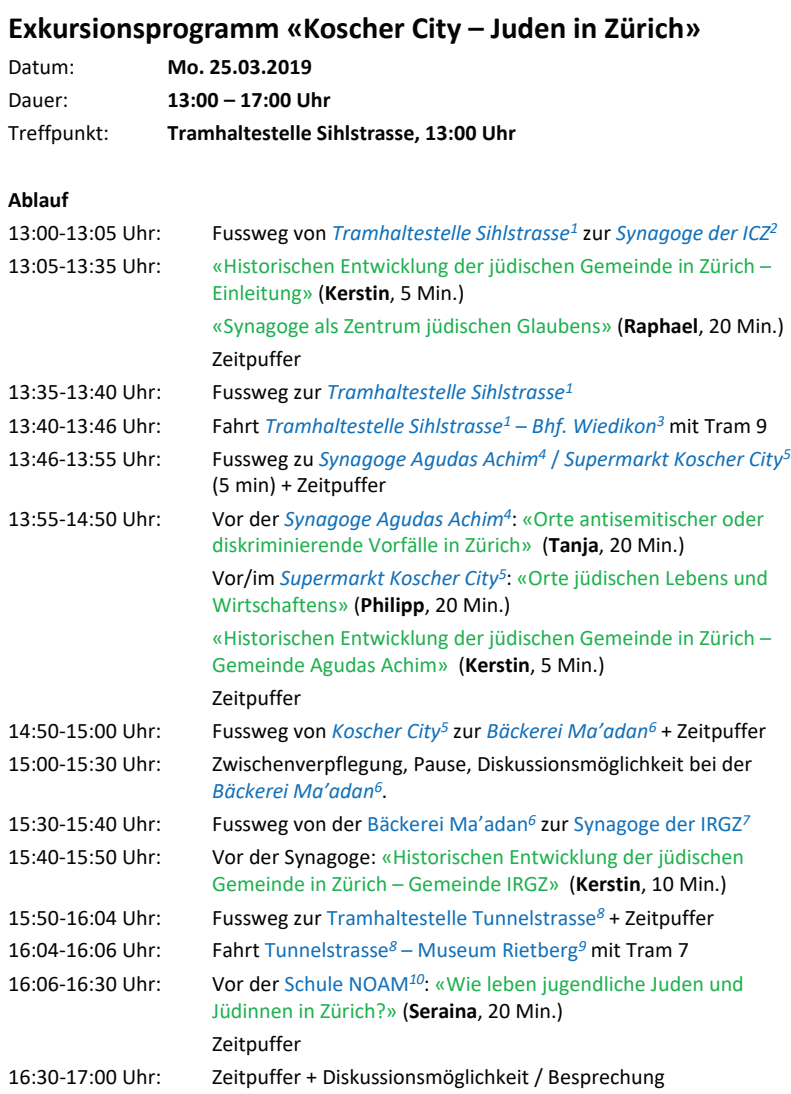

Die erste Phase des Seminars sah eine inhaltliche Einarbeitungszeit vor. Die Ergebnisse trugen die Studierenden zu einem thematischen Rundgang durch das jüdisch geprägte Zürcher Stadtviertel Wiedikon zusammen, der in der nachfolgenden Karte detailliert aufgezeigt wird. Während des Rundgangs erklärten sich die Studierenden - aufbauend auf ihren thematisch gewählten „Lernorten“ - gegenseitig vielfältige Facetten des jüdischen Viertels und ihrer Bewohner/ innen (siehe Karte und Programm unten) und legten somit eine gute Wissensbasis für die zweite Phase des Seminars.

Die zweite Phase des Seminars war dem Projekt "Likrat“ (hebräisch für „aufeinander zu“) gewidmet, wobei wir einen Schwerpunkt auf die Schweiz bzw. Zürich setzten. Dialogprojekte des Netzwerkes „Likrat" gibt es mittlerweile in ganz Europa und werden von den jeweiligen jüdischen Vereinen und Organisationen (z. B. dem Zentralrat der Juden in Deutschland) gefördert. In der Schweiz wird Likrat vom Schweizer Israelitischen Gemeindebund (SIG) unterstützt und schult junge Jüdinnen und Juden (ab ca. 15 Jahren), damit sie jeweils zu zweit Schulbesuche in der Schweiz durchführen können. Ein Ziel besteht darin, dass in offenen Gesprächskreisen unter Jugendlichen tabufrei heikle Themen diskutiert werden. Damit soll ein wichtiger Beitrag zur Prävention von Rassismus

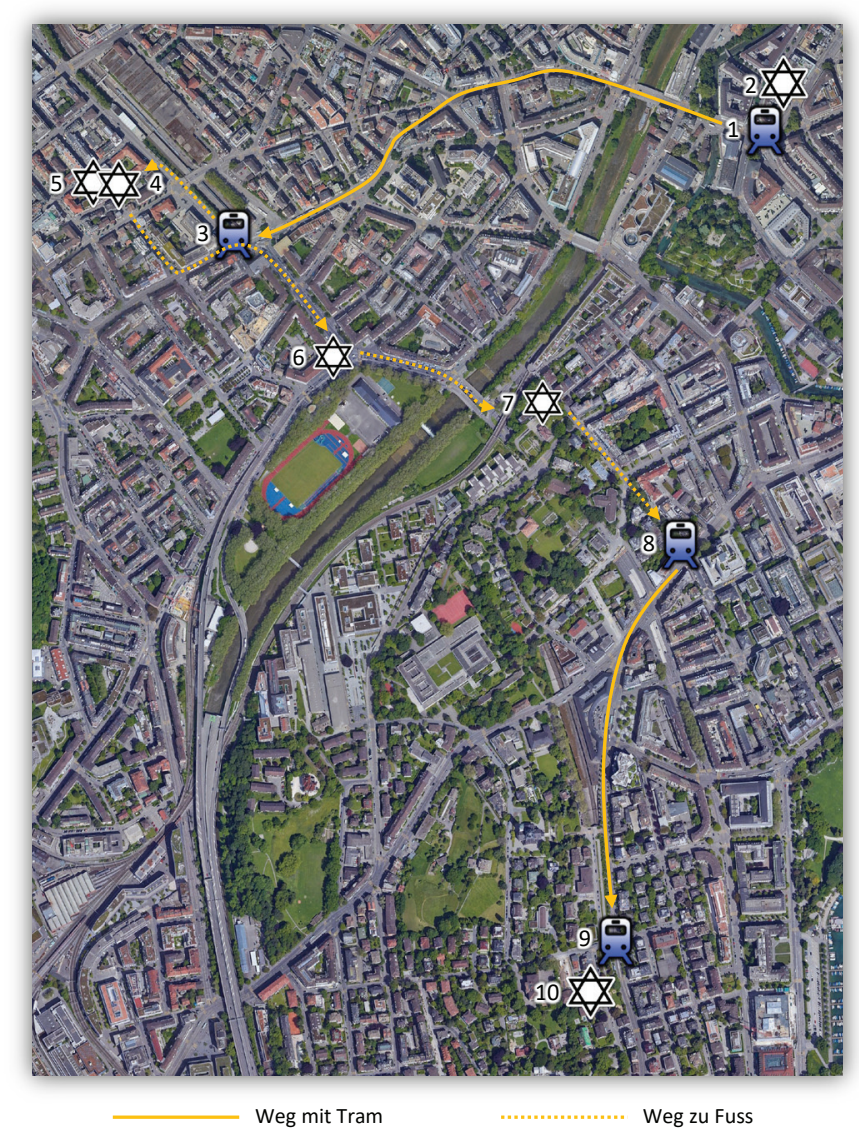


und Antisemitismus geleistet werden. Einen sehr guten Einblick in die Arbeit der Likrati-Jugendlichen vermittelt der Kurzfilm, der auch auf der Startseite von likrat.ch veröffentlich ist: https://www.likrat.ch/ de/schulen/

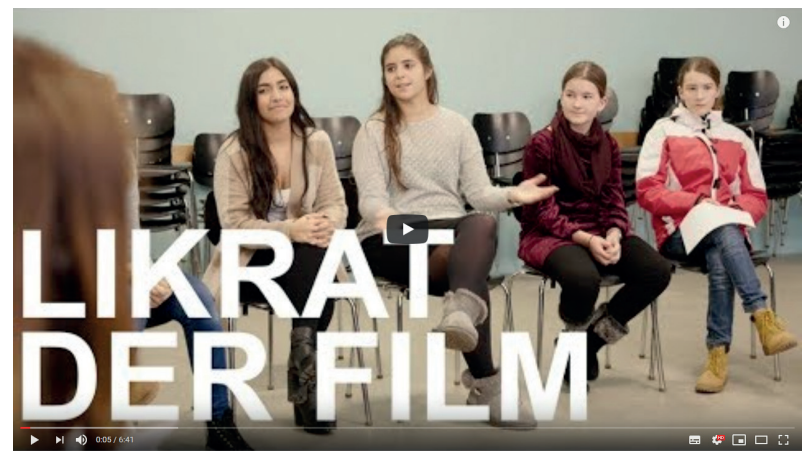

Abb. 2: Screenshot Likrat

(Quelle: https://www.likrat.ch/del, 17.04.2019)

\section{Kontextualisierung: Jüdinnen und Juden in der Schweiz}

Von den sieben Millionen Einwohner/innen der Schweiz, die im Bezugsjahr 2017 älter als 15 Jahre sind, stellen die ca. 18000 Schweizer Jüdinnen und Juden mit einem Bevölkerungsanteil von $0.3 \%$ nur eine kleine (religiöse) Minderheit dar (Bundesamt für Statistik 2019). Die Geschichte der jüdischen Minderheit blickt auf eine lange Migrationsgeschichte zurück, die bis ins 12. Jahrhundert zurückreicht (Guggenheim-Grünberg 1967; Historisches Lexikon der Schweiz o.J.). Antisemitische Vorurteile (z.B. Juden würden die Brunnen vergiften und die Pest in die Stadt bringen) und Vorfälle waren auch in der Schweiz weit verbreitet und wurden von der christlichen Kirche weiter verstärkt (Brunschwig et al. 2005). Bei einem Pogrom in der Nacht am 24. Februar 1349 wurden Zürcher Jüdinnen und Juden in ein Haus eingesperrt, das daraufhin angezündet wurde (Girardet 2001). Während Brunnenvergiftungen als Vorwand angegeben wurden, lagen die Beweggründe für dieses Pogrom aber eher in finanziellen Angelegenheiten: Nach der Ermordung der Jüdinnen und Juden wurden sämtliche Schulden, die Christinnen und Christen bei ihnen gehabt hatten, für nichtig erklärt. Auch die Stadt Zürich bereicherte sich am Erbe der Verbrannten (Brunschwig et al. 2005; Ruoff et al. 1995).

Während im späten Mittelalter Jüdinnen und Juden vor allem auf dem Land (z. B. im aargauischen Surbtal, Endingen und Lengau) angesiedelt waren und je nach gesellschaftlicher Lage von Städten entweder angelockt oder verstoßen wurden, so erlangten Jüdinnen und Juden in der Schweiz erst im Jahr 1874 volle Bürgerrechte, was Niederlassungsfreiheit und Berufs- freiheit miteinschloss. In dieser Zeit wurden viele bekannte Synagogen, z. B. die Synagoge an der Löwenstrasse in Zürich (1880-1884), gebaut. Als Ergebnis der ersten Initiative auf Bundesebene überhaupt, wurde 1894 das Schächtverbot in die Bundesverfassung aufgenommen, das zuvor einen Grundsatzstreit zwischen Religionsfreiheit und Tierwohl ausgelöst hatte. Der große Erfolg der Initiative wurde auch mit der antisemitischen Haltung großer Bevölkerungsteile in der Schweiz erklärt (Longchamp et al. 2000). Im Jahr 1978 wurde das Schächtverbot aus dem Grundgesetz gestrichen und inhaltlich durch das Tierschutzgesetz ersetzt, das auch Muslime in der Schweiz betrifft. Der bislang letzte Versuch das de facto Schächtverbot zu lockern scheiterte 2001 (Dreyfuss-Kahn 2013: 85-6).

In der Gegenwart befinden sich die zwei größten jüdischen Gemeinden in Genf (3500) und Zürich (5500) (Statista 2019), wobei hinter diesen Zahlen ein sehr heterogenes Spektrum von "Jüdisch-sein" steckt. In einer vom Schweizer Nationalfonds geförderten Studie zum „Schweizer Judentum im Wandel“ (Gerson et al. 2010) werden als die drei zahlenmäßig bedeutendsten religiösen Strömungen innerhalb der jüdischen Glaubensrichtung unterschieden:

- Orthodox: Dieser Richtung fühlen sich die meisten Einheitsgemeinden im Schweizerischen Isrealitischen Gemeindebund (SIG) verpflichtet; darunter auch die Israelitische Cultusgemeinde Zürich (ICZ).

- Ultraorthodox/Charedisch: Die Zürcher SIG-Gemeinde Israelitische Religionsgemeinschaft (IRG) und Agudas Achim zählen beispielsweise hierzu.

- Liberal/Reform: Hierzu wird u. a. die Jüdisch Liberale Gemeinde Or Chadasch (JLG) gezählt.

Diese drei Glaubensrichtungen, die intern weiter differenziert werden können, sind auch in der Stadt Zürich vertreten. Für die jüdische Gemeinde waren verschiedene Einwanderungswellen im Laufe der Geschichte bedeutend, wobei insbesondere Jüdinnen und Juden aus osteuropäischen Ländern im Verlauf des 20. Jahrhunderts die ultraorthodoxe jüdische Gemeinde vergrößerten.

Religion ist ein Aspekt des gesellschaftlichen Lebens, der einem starken Wandel bzw. Bedeutungsverlust, insbesondere unter jüngeren Bevölkerungsgruppen, unterliegt. Waren in der Stadt Zürich 1850 noch über $90 \%$ der Bevölkerung evangelisch-reformiert, so ist die größte Gruppe heute die der Konfessionslosen (36.4\%), gefolgt von römisch-katholischen (26.7\%), evangelisch-reformierten (21.1\%) und muslimischen (5.8\%) Zürcherinnen und Zürchern. Zum jüdischen Glauben bekennen sich nur 1.1\% der Stadtbevölkerung, der landesweite Durchschnitt liegt bei $0.3 \%$ (Bundesamt für Statistik 2019).

In den Zürcher Kreisen 2 (Enge, Wollishofen, Leimbach) und 3 (z. B. in Wiedikon) stellen Jüdinnen 


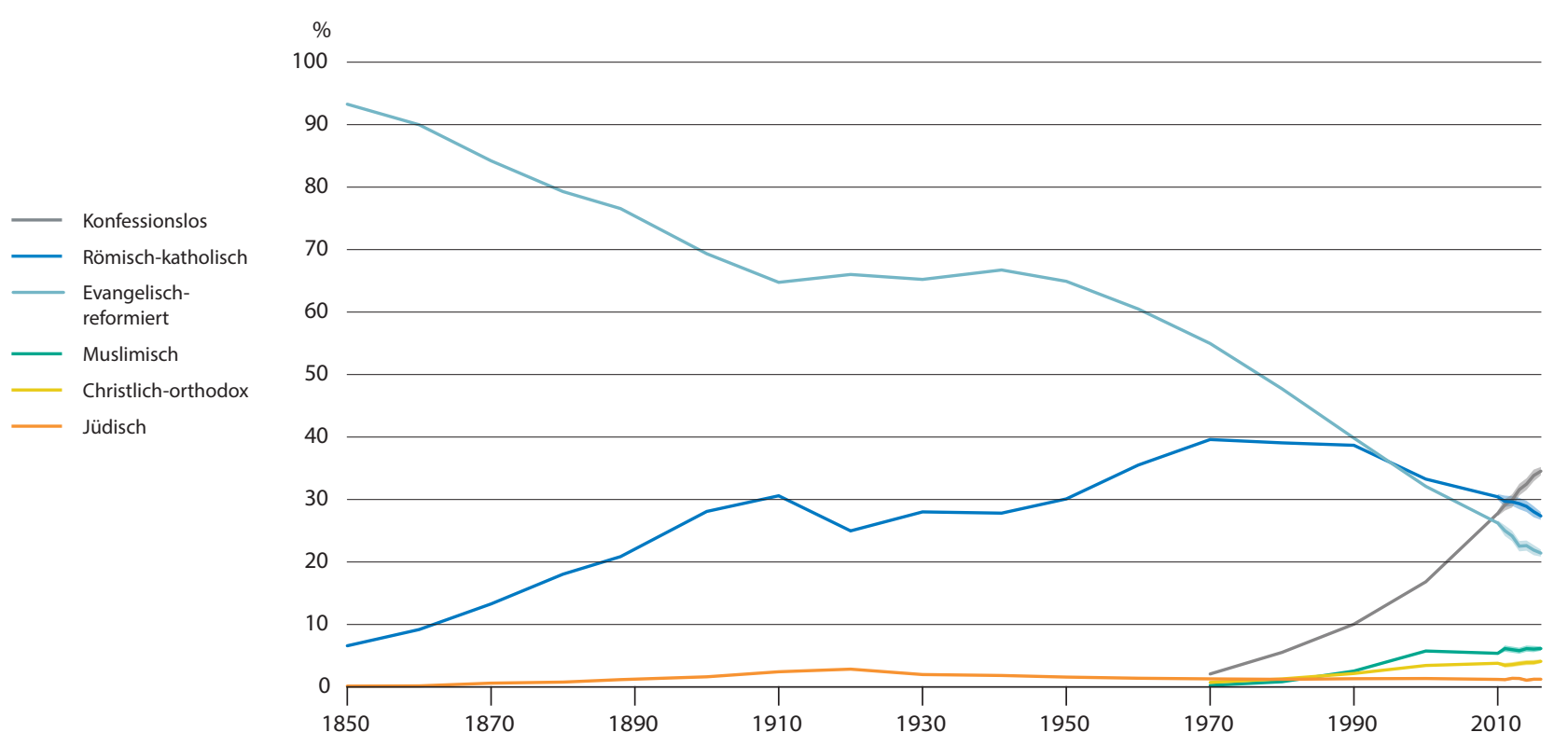

Abb. 3: Stadtzürcher Religionslandschaft (Quelle: Statistik Stadt Zürich)

und Juden z.T. bis zu $10 \%$ der Quartiersbevölkerung dar (vgl. Stadtzürcher Religionslandschaft 2019). Diese punktuellen Segregationsphänomene können, ebenso wie die politische Einstellung, das Alter und die Alltagserfahrungen der Befragten, einen Anhaltspunkt für die Ergebnisse einer Studie aus dem Jahr 2000 zur Einstellung der Schweizer/innen gegenüber Jüdinnen und Juden und dem Holocaust geben. Die Resultate zeigten, dass die Befragten den Anteil von Jüdinnen und Juden an der Bevölkerung mehrheitlich überschätzten. So mutmaßten etwa $41 \%$ der Befragten, den Anteil von Jüdinnen und Juden an der Gesamtbevölkerung der Schweiz zwischen 1\% und 9\%. Weitere $13 \%$ der Befragten wähnten diesen Anteil auf $10 \%$ bis $19 \%$, $5 \%$ der Befragten gingen von einem noch höheren Anteil aus und etwa ein Drittel hatte gar keine zahlenmäßige Vorstellung. Nur 6\% lagen mit ihrer Einschätzung (weniger als $1 \%$ der Bevölkerung) richtig (Antisemitismusbericht 2000: 16).

Als die größte Stadt in der Schweiz ist Zürich sicher ein besonderes soziales Gefüge in der Schweizer Gesellschaft. Derzeit leben in der Stadt Zürich Menschen aus 171 Nationen zusammen. Der Ausländer-/ innenanteil liegt bei 32.4\% (2017) (Statistik Stadt Zürich 2019). Wichtig ist darüber hinaus festzuhalten, dass ca. $75 \%$ der jüdischen Bevölkerung einen Schweizer Pass hat (Weill 2004: 120-129).

Zürich ist demnach eine Stadt, in der Migration ein sichtbarer Teil der Geschichte und des Alltagslebens ist. Christiane Hintermann und Dirk Rupnow (Hintermann 2019; Hintermann \& Rupnow 2016) haben an lokalen Beispielen in Österreich sehr schön herausgearbeitet, dass Migrationsgeschichte und Geschichte/n von Minderheiten in der Geschichts- schreibung und im Geschichtsbewusstsein der Majoritätsgesellschaft Gefahr läuft, eine eher vernachlässigte Erzählung der Nationalgeschichte zu sein.

Die Geschichte der jüdischen Minderheit bzw. die Migrationsgeschichte von Jüdinnen und Juden in die Schweiz ist ein besonderes Kapitel der Schweizer $\mathrm{Na}-$ tionalhistorie, wie die Ausführungen am Anfang des Kapitels zeigten. Ein Kapitel, das eng mit der Rolle der Schweiz im Zweiten Weltkrieg bzw. der Migration von Jüdinnen und Juden in die Schweiz verbunden ist. Dieser Tatsache wird auch in den Schulen Rechnung getragen. Mit der Einführung des Lehrplans 21 (Grundlehrplan für die Lehrpläne in Primar- und Sekundarschulen in 21 Schweizer Kantonen) vor wenigen Jahren wurde festgelegt, dass ein sehr vielfältiges Bild der Schweizer Nationalgeschichte und der Gegenwartsgesellschaft erlernt werden soll. So wird etwa die Nationalgeschichte der Schweiz im Fach „Räume, Zeiten, Gesellschaften“ (RZG), gemäß dem Lehrplan 21, anhand von wichtigen Ereignissen der Schweizer Geschichte im 20. Jahrhundert vermittelt, wobei hier als verbindliche Themen u. a. „die Schweiz während der Zeit der Weltkriege“, „Landesstreik“ und "Frauenstimmrecht" gesetzt sind (www.lehrplan21.ch, RZG 5.1). Im Fach „Religionen, Kulturen, Ethik“ (RKE) hingegen lernen die Schüler/innen an der Geschichte des Judentums in der Schweiz und Europa Erfahrungen religiöser und kultureller Minderheiten exemplarisch aufzuzeigen (Diaspora, Identität, Toleranz, Emanzipation, Antisemitismus, Schoah/Holocaust, öffentliche Anerkennung, Zionismus) (www. lehrplan 21.ch, RKE 3.2). Beide Aspekte, also sowohl die Geschichte der Schweiz während des Zweiten Weltkriegs als auch die jüdische Gemeinschaft als re- 
ligiöse Minderheit in der Schweizer Mehrheitsgesellschaft, werden in den allgemeinbildenden Schulen verbindlich behandelt. Im Lehrplan 21 sind diese beiden Themen als gut miteinander verknüpfbare Inhalte und mit entsprechenden Querverweisen aufgeführt. Auch an Schweizer Gymnasien sind Themen wie Migration und Minderheiten ein fester Bestandteil des Geographie-Curriculums (z. B. Lehrplan Geographie Kantonsschule Zürich-Nord).

Das Thema jüdisches Leben bietet sich gerade in Städten wie Zürich, Wien oder Berlin an, weil hier vor Ort historische und geographische Aspekte anschaulich zusammengeführt werden können. Vor diesem Hintergrund habe ich auch die provokativen Thesen und Argumente von Max Czollek zum Anlass genommen, gemeinsam mit einer Gruppe von fünf Lehrdiplom-Studierenden im Rahmen des beschriebenen Moduls die Frage zu stellen, wie es denn um das "Gedächtnistheater" in der Schweiz und die gesellschaftliche Positionierung von Jüdinnen und Juden hier bestellt ist. Natürlich sind hier die Aufarbeitung der Geschichte und die Schuldfrage ganz anders gelagert als in Deutschland. Aber der Mythos der „neutralen Schweiz“ kann aufgrund ihrer dokumentierten Finanz- und Wirtschaftsbeziehungen zum Dritten Reich und ihre z.T. antisemitische Flüchtlingspolitik nicht wirklich aufrechterhalten werden (vgl. Bergier et al. 2002). Erst sehr spät - Mitte der 1990er Jahre - setzte sich die Schweizer Öffentlichkeit mit der Frage auseinander was ihre Verantwortung in Bezug auf die Shoa während des Zweiten Weltkriegs und die Behandlung von jüdischen Wertanlagen in der Nachkriegszeit anbelangte (Bergier et al. 2002). 1995 wurde in New York im Namen des Jüdischen Weltkongresses eine Sammelklage eingereicht. Die Kläger behaupteten, Holocaust-Überlebende hätten keinen Zugriff auf ihnen zustehende Bankkonten in der Schweiz.

„Im Juli 1997 veröffentlichten die Schweizer Banken eine neue Liste mit nachrichtenlosen Vermögen.

Die Liste erschien in Zeitungen auf der ganzen Welt.

(...) Am 12. August 1998 schlossen die Schweizer Banken UBS und Credit Suisse einen Vergleich mit dem World Jewish Congress. Die Banken verpflichteten sich in diesem 1995 in New York eingereichten Gerichtsverfahren, 1,16 Mrd. Franken (1,25 Mrd. \$) an Holocaust-Überlebende und ihre Nachkommen auszuzahlen. Davon wurden 742 Mio. Fr. (800 Mio. \$) für Personen vorgesehen, deren Gelder nach dem Krieg auf Konten in der Schweiz liegengeblieben waren. Weitere 394 Mio. Fr. (425 Mio. \$) waren reserviert für Holocaust-Überlebende, an der Schweizer Grenze abgewiesene Flüchtlinge und Zwangsarbeiter. Auch nichtjüdische Opfer erhielten Zahlungen." (Wurz 2013)
Neben den Reparationszahlungen - die sich noch bis ins Jahr 2013 hinzogen, weil die Banken die Identität der Kontoinhaber/innen und ihrer Nachfahren z.T. nur schwer ausfindig machen konnten setzte das Schweizer Parlament 1996 eine unabhängige Kommission ein, die die Rolle des Finanzplatzes Schweiz während des Zweiten Weltkriegs aufarbeiten sollte. Unter dem Vorsitz des Schweizer Historikers Jean-François Bergier, zeigte der sogenannte „Bergier-Bericht“ (Bergier et al. 2002) detailliert die komplexen Wirtschaftsbeziehungen zwischen der Schweiz und dem NS-Regime auf. Auch die Flüchtlingspolitik der Schweiz wurde dort kritisch erörtert: „Gemessen an ihren vormals humanitären und asylpolitischen Vorstellungen, verfehlte die neutrale Schweiz in der Flüchtlingspolitik nicht nur den selbstgesteckten Standard, sondern verstieß auch gegen elementare Gebote der Menschlichkeit" (Bergier et al. 2002: 523).

Einen weiteren Meilenstein in der etwas anderen "Schweizergeschichte“ markierte der Historiker Thomas Maissen (2005), indem er die Schweizer Öffentlichkeit erneut dazu brachte sich mit diesem unangenehmen Thema auseinanderzusetzen. In seinem Buch „Verweigerte Erinnerung“ machte er unmissverständlich klar, dass die Schweizer Banken eine Rückgabe der „herrenlosen Vermögen“ verstorbener Jüdinnen und Juden nach dem Zweiten Weltkrieg nicht nur verzögerten, sondern diese sogar „zielstrebig sabotiert“ hätten (Maissen 2005: 55).

„Sehr bewusst unterliessen sie nicht nur jede eigene Initiative, sondern sabotierten zielstrebig so lange, wie es möglich war, auch eine entsprechende (Sonder-)Gesetzgebung, die allein der außergewöhnlichen Situation nach der Shoah hätte angemessen sein können. "(Maissen 2005: 55)

Eine "Geste der Entschuldigung" hätte Maissen ebenso angebracht gefunden, wie dafür zu sorgen, dass diese historischen Fakten im Bewusstsein der breiten Bevölkerung verankert werden. In diesem Sinne war es Maissen ein Anliegen, sich nachhaltig und schonungslos mit der eigenen Vergangenheit auseinanderzusetzen: „Nur ein Volk, das den Zweiten Weltkrieg selbst schadlos überstanden hatte, konnte sich der Erinnerung der Opfer auf eine Art verweigern, wie viele Schweizer dies zumindest unbewusst taten" (Maissen 2005: 29).

Im Zusammenhang mit der relativ späten Aufarbeitung der Frage, welche Rolle die Schweiz während und nach dem Zweiten Weltkrieg in Bezug auf die Shoa spielte, kann auch der Schlussbericht des SNFProjekts zum Thema „Schweizer Judentum im Wandel" (Gerson et al. 2010) gesehen werden, dem dieser Beitrag eine fundierte thematische Einführung und eine verlässliche Zahlenbasis verdankt. 


\section{4 „Gedächtnistheater“ und „Desintegriert euch!" aus Sicht der Studierenden und der jüdischen Jugendlichen}

Die Studierenden und ich begleiteten im Mai 2019 einen Schulbesuch von zwei Likratinos an eine Sekundarschule (Progymnasium) in Aarau (AG) als teilnehmende Beobachter/innen und führten im Anschluss daran noch eine einstündige Diskussion mit den zwei Jugendlichen. Im Gespräch mit Yael (19) und Aaron² (17), ging es sowohl um den Austausch der Beobachtungen während des Schulbesuchs, als auch um die Diskussion des Schweizer "Gedächtnistheaters" und um die Rolle von jugendlichen Jüdinnen und Juden in der Schweizer Gesellschaft, was wir in Verbindung brachten mit der "Desintegrationsthese“ von Max Czollek.

In der Seminarstunde vor dem Schulbesuch überlegten wir zusammen, wie wir den provokativen Inhalt des Buches „Desintegriert euch!“ (Czollek 2018b) für die zwei Likratinos, die dieses Buch nicht kannten, in einer angemessenen Form zusammenfassen und veranschaulichen könnten. Folgende Thesen schrieben wir auf Flipcharts und legten sie vor dem Gespräch auf dem Boden aus:

- Welche Rolle spielen Jüdinnen und Juden in der Schweizer Geschichte?

- Sind Jüdinnen und Juden zu „passiv“ in der Vertretung der eigenen Geschichte und Interessen?

- These Czolleks: Jüdinnen und Juden sollten selbstbewusster und provokativer für ihre eigene Geschichtswahrnehmung und ihre Gegenwartsanliegen eintreten!

Obschon der inhaltliche Hintergrund dieser Fragen im Zentrum des offenen Austausches stand, behandelten wir diese Fragen nicht in chronologischer Reihenfolge. Diese Vorgehensweise wäre viel zu statisch gewesen für einen Austausch unter fast Gleichaltrigen. Dementsprechend konnte sich das Gespräch auch sehr dynamisch entwickeln und fand in einer lockeren Atmosphäre statt. In den folgenden Abschnitten habe ich die im Gespräch vorgebrachten Argumente der Likratinos und der Studierenden ausgewertet und unter den zwei Leitthemen „Schweizer ,Gedächtnistheater“" und „Desintegriert euch!" detaillierter ausgearbeitet. Hierdurch tritt die Perspektive von Jugendlichen zu den zentralen Themen stärker in den Vordergrund und erweitert die inhaltliche Debatte des Artikels um persönliche Sichtweisen.

\subsection{Gedächtnistheater}

Anhand von aufgezeichneten Diskussionsausschnitten möchte ich auf zwei Aspekte des Gedächtnisthea-

Namen geändert ters eingehen. Erstens, die von der Weltmeisterschaft 2006 markierte „Normalisierungsthese“ in Bezug auf das deutsche Nationalgefühl und zweitens die Sichtweise von Yael auf das „Schweizer, Gedächtnistheater“".

Aus der Diskussion während des Vorbereitungstreffens entwickelte sich ein interessanter Austausch unter den Seminarteilnehmern/innen. Vor allem einen Studenten beschäftigte der Gegenwartsbezug des Gedächtnistheaters ganz besonders: ${ }^{3}$

Student A: Ja, ich fände das schon auch spannend $z u$ hören ob sie (= Likrati-Jugendliche) damit ein Problem haben, dass die heutige Generation Jugendlicher einen Nationalstolz verspüren, weil er (= Max Czollek) stört sich extrem an der Fussball-WM. Und er sagt, er redet ausschließlich von Nationalismus. Aber Nationalismus impliziert ja, dass man seine eigene Nation über eine andere stellt. Und das ist in einem sportlichen Sinne für mich ein bisschen krass formuliert. Ich meine, ich bin auch Fußball-Fan und freue mich, wenn die Schweiz gegen ein anderes Land gewinnt (lacht), aber das ist für mich nicht Nationalismus. Jo. Eher Patriotismus, vielleicht, na, auch nicht zwingen, aber ich finde dieses FußballBeispiel, ich störe mich da ein wenig dran, dass er das, ich weiß nicht, dass die heutige Generation gar nicht darf.

Studentin B: Ja, es geht ja auch um die Flagge-

Student A: Flagge, Hymne-

Studentin B: Er hat dann etwa das Gefühl, so, die, wie kann dann ein jüdischer Jugendlicher sich darüber freuen und die deutsche Flagge schwingen, oder? Irgendwie, es geht um die Frage mit dem Hintergrund des ganzen Nationalsozialismus und Shoa und so.

Der Student A stört sich insbesondere an Czolleks "Normalisierungsthese“. Diese beinhaltet, dass in Deutschland während der Fußball-Weltmeisterschaft im Jahr 2006, an dessen Ende Deutschland den 3. Platz belegte, ein neues bzw. lang verborgenes $\mathrm{Na}$ tionalgefühl des „Endlich dürfen wir wieder“ (Czollek 2018b: 38) zelebriert wurde. Etwas polemisch und übergeneralisierend stellt er fest, dass „(d)ie Deutschen die WM 2006 als eine kollektive Erleichterung darüber (erlebten), dass es endlich wieder erlaubt war, die Deutschlandfahne zu schwingen wie früher. Das ist es was ich ,Täter*innengemeinschaft' nenne“ (Czollek 2018b: 38). Der Student bezieht die Normalisierungsthese zwar auf sich als Fußballfan, in seiner Rolle als „Schweizer“ bleibt er hingegen historisch unangreifbar und neutral. Für ihn ist es wohl nicht ganz einfach nachzuvollziehen, wie ambivalent das Verhält-

Die Namen der Studierenden habe ich auf ihren Wunsch hin in den Interviewausschnitten anonymisiert. 
nis der deutschen Nachkriegsgesellschaft bis dahin zu allen Bezügen des eigenen „Nationalen“ (z. B. Nationalhymne) war. Bei Max Czollek ist die weiter oben erläuterte „Normalisierungsthese“ eng verknüpft mit dem „Integrationsparadigma“: „Wenn ich vom Integrationsdenken oder vom Integrationsparadigma schreibe, dann meine ich die Konstruktion eines kulturellen und politischen Zentrums, das sich implizit oder ausdrücklich als deutsch versteht" (Czollek 2018a: 15; Herv. i.O.). Die Forderung nach „Desintegriert euch!" richtet sich im Buch jedoch häufig explizit an Jüdinnen und Juden - verbunden mit der Aufforderung, die eigene Geschichtsdarstellung offensiver und die vielfältige Gegenwartsgesellschaft nachdrücklich zu vertreten. Nicht die einzelnen Argumente selbst, sondern vielmehr ihre Verschränkung miteinander, also das Ausspielen einer nationalen gegen eine religiöse Kategorisierung, wurde von den Studierenden im Seminar kritisch beurteilt.

In einem geschichtlichen Kontext, der aus einem anderen Blickwinkel das „Gedächtnistheater" beleuchtet, stellen zwei Studentinnen an Yael und Aaron die Frage, wie sie die Rolle der Schweiz im Zweiten Weltkrieg in Bezug auf Jüdinnen und Juden sehen.

Studentin C: (...) "das Boot ist voll" und die ganze Geschichte aus dem Zweiten Weltkrieg, Gewisse, die es nicht geschafft haben, Gewisse, die geholfen haben aus der Schweiz, ich glaube die Idee vom Stern oder vom J im Pass ist glaube ich aus der Schweiz gekommen, das habe ich mal gehört.

Studentin D: Ja, das habe ich auch so gehört.

Studentin C: Ich glaube die Schweizer haben gesagt, sie wüssten gar nicht wer Jude ist und daher wollten sie das im Pass so sehen.

Yael: Ich glaube aber auch, dass weil die Schweiz im Zweiten Weltkrieg eine ziemlich kleine Rolle gespielt hat und sich nicht wirklich hat beteiligen wollen und dann doch gewisse Sachen gelaufen sind, dass man heute weiß, sie haben Transporte erlaubt oder Gewisse abgelehnt, Gewisse nicht, glaube ich, dass es für Schweizer Juden schwierig ist zu wissen, wie die Schweiz zu ihnen steht. Also, man weiß heutzutage schon, dass die Schweiz zu allen schaut und so, und auch Juden in der Schweiz friedlich leben können.

In diesem Ausschnitt wird das „Schweizer Gedächtnistheater" zumindest ansatzweise angeschnitten. Yael verweist darauf, dass die Schweiz zwar nur eine sehr geringe Rolle im Zweiten Weltkrieg gespielt hat, allerdings auch nicht ganz frei von Mittäterschaft und Mitschuld gesprochen werden kann. Besonders bemerkenswert finde ich an ihrer Aussage, dass sie nicht wisse, wie die Schweiz zu Schweizer Juden stehe. Diese Unsicherheit ist sehr gut nachvollziehbar im Anblick der historischen Tatsachen (z. B. Eintrag als Jüdin/Jude im Reisepass). Allerdings hat Yael diese
Aussage im Präsens formuliert, was vielleicht darauf hindeutet, dass diese Frage für sie eine/n konkrete/n Gegenwartsbedeutung und -bezug hat. Wir haben an dieser Stelle im Gespräch nicht weiter nachgehakt, sodass ich hinter diese Interpretation ein Fragezeichen setzen möchte.

Aaron erzählt in Zusammenhang mit der Geschichte der Jüdinnen und Juden während des Zweiten Weltkriegs und dem Erinnern an den Holocaust, dass er erst vor einiger Zeit an einer Veranstaltung der „International March of the Living"-Organisation teilgenommen hat. Auf dem gemeinsamen Weg von Auschwitz nach Birkenau habe er die wertvolle Erfahrung und Bekanntschaft mit Holocaust-Überlebenden gemacht. In diesem Sinne habe er sich also zu einem Teil des "Gedächtnistheaters" machen lassen. Sich und andere aber weiterhin an die Geschichte der ermordeten, misshandelten und vertriebenen Jüdinnen und Juden zu erinnern, ist ihm persönlich ein Anliegen und Grund genug sich bei Likrat zu engagieren. Zum Nachdenken über das „Schweizer Gedächtnistheater" hat uns im Seminar angestoßen, dass es zwar einen internationalen Shoa-Gedenktag gibt, der jedes Jahr am 27. Januar an die Befreiung des KZ Auschwitz-Birkenau im Jahr 1945 erinnert. Entsprechende räumliche Analogien, also Orte des Shoa-Gedenkens, sind in der Schweiz jedoch äußerst rar gesät, abgesehen vom Archiv für Zeitgeschichte der ETH Zürich und der Gedenkstätte für jüdische Flüchtlinge in Riehen bei Basel.

\section{2 „Desintegriert euch!“}

Yael: (...) Ja, ich glaube schon, es gehört schon zum Stadtbild von Zürich, oder zumindest von bestimmten Quartieren, dass hier viele Juden leben. Ja, ich glaub jetzt nicht, dass die Rolle so passiv ist, weil wir halt doch so wenige sind im Verhältnis, denke ich schon, dass es schwierig ist sich megafest einzubringen. Aber so Sachen wie das jüdische Filmfestival, das es jetzt auch schon einige Jahre gibt, mein Vater ist daran auch beteiligt. Da zeigt man ja doch auch viel Präsenz und auch was einen auch viel beschäftigt mit diesen Filmen und sonst, $j a$, weiß nicht wie gut es wäre, wenn wir noch viel mehr Präsenz zeigen würden. Ich glaube da gäbe es sicher Leute, die es nerven würde oder die das nicht richtig fänden. (...) Also, man weiß heutzutage schon, dass die Schweiz $z u$ allen schaut und so, und auch Juden in der Schweiz friedlich leben können. Aber trotzdem gibt es Leute, die antisemitische Gedanken haben, das weiß man auch und dass man darum genau auch wenig Präsenz zeigen will. Weil man weiß, mit jeder Präsenz kann man auch Leute provozieren, die gegen Juden sind. Und daher, auch wenn es ein Event ist, 
ein Gedenktag oder so, braucht es für uns Sicherheit trotzdem am Ende vom Tag und es gibt immer wieder Bedrohungen auch gegen die jüdische Gemeinde, z. B. Bombendrohungen gegen das jüdische Zentrum und dann, ja, frage ich mich schon: Sollen wir noch mehr Präsenz zeigen? Das kann ja auch gefährlich sein!

Yael konfrontiert die provokativen Forderungen Czolleks nach mehr Präsenz und aktiver Teilhabe an der Vergangenheitsbewältigung und Gegenwartsgestaltung des öffentlichen Lebens mit einem wichtigen Gegenargument. Obschon sich Yael grundsätzlich sicher in Zürich fühlt, sind ihre Bedenken gegenüber Angriffen auf Jüdinnen und Juden durchaus berechtigt, wie etwa ein Vorfall aus dem Sommer 2018 belegt:

Mann verfolgt in Zürich drei Juden mit einem Messer Am Samstagabend hat ein Vorfall auf offener Strasse im Zürcher Quartier Wiedikon für Aufregung gesorgt. Ein Mann starrte zuerst eine Gruppe jüdischorthodoxer Kinder auf dem Spielplatz an. Als etwas später drei Gläubige zur Synagoge aufbrechen, werden sie vom selben Mann verfolgt. Er hat plötzlich ein Messer in der Hand und brüllt antisemitische Parolen. Der Angreifer konnte von einem Passanten gestoppt werden. (Tagesanzeiger 09.07.2018)

Auch sie selbst hat schon Erfahrungen mit unangenehmen Situationen gemacht oder wurde zusammen mit ihren Freundinnen als "Jüdin“ auf offener Straße beschimpft. Solche und ähnliche antisemitischen Vorfälle werden jedes Jahr vom Schweizerischen Israelitischen Gemeindebund (SIG) erfasst und registriert, wobei dieser eine deutliche Verschiebung der Angriffe und „Hate Speech“, insbesondere in Internet-Publikationen und sozialen Netzwerke, registriert (siehe Antisemitismusbericht 2018; https://www.swissjews.ch/ de/antisemitismus /vorfall-melden/, 21.3.2019)

Im Gespräch mit Aaron und Yael werden noch weitere Themen erörtert, etwa ihre familiären Beziehungen zur Shoa, die Bedeutung von Religion im Leben von jüdischen Jugendlichen sowie die Problematik der inneren Spaltung der jüdischen Gemeinschaft in unterschiedliche Gruppierungen. Gerade dieser Punkt ist der Auslöser für ein Streitgespräch zwischen den beiden Jugendlichen:

Aaron: Also ich finde, es ist ja schon generell ein bisschen crazy, dass es eigentlich wenige Juden in der Schweiz hat, aber eher noch mehr in Zürich. Und dass man sich trotzdem in drei Gemeinden aufspaltet von denen. Jede Gemeinde macht sich über die andere lustig. Ahm, ich bin im Jugendbund, das ist wie Pfadi und auch nachher ein Teil vom Leben. Ich gehe dort schon hin seit ich sechs bin, jeden Samstag von drei bis sechs Uhr und wir haben es geschafft, es gibt drei Jugendbünde, die sich im gleichen Gebäude zur gleichen Zeit treffen und ahm, es hat solche, die sind nicht religiös, eher zionistisch und dann halt meinen Jugendbund, der ist eher traditionell, und dann hat es noch (unverständlich) und wir nehmen uns eigentlich so gegeneinander die Kinder weg.

Itta: Ja, ja.

Aaron: Und ich finde es schade, dass wir es nicht schaffen, die zusammen zu bringen.

Yael: Ja, aber am Schluss gibt es doch für jeden etwas in den Gruppen-

Aaron: Ja, sicher-

Yael: In dem Jugendbund, wo er jetzt ist war ich auch früher. Und dann habe ich gemerkt, dass es wie nicht meines ist. Ich wollte etwas Religiöseres. Und da hat es einfach für mich gestimmt. Und drum finde ich es eigentlich schön, dass es wie in einem Haus die Möglichkeit gibt verschiedene Angebote zu haben.

Aaron: (fällt ins Wort) Ja, aber man läuft sich ja schon über den Weg und wenn man den anderen sieht, ist es ein scheues Hallo, aber dann geht man zurück in seine Gruppe. Und es sind ja doch immerhin 150, 200 Jugendliche, die dann in dieses Haus kommen aber nichts miteinander wirklich machen. Das finde ich persönlich fast ein bisschen schade. Nur weil die jeweiligen Eltern vielleicht etwas weniger religiös sind als die anderen.

Yael: Und es gibt ja auch nichts, was man irgendwie kombinieren könnte oder so.

Aaron: Doch, also in Deutschland hat es eine massive Organisation, wo alle zusammenkommen.

Yael: Ja, aber wenn jemand religiös ist und würde dorthin gehen, und nicht die Regeln befolgen, und möchte Teil unserer Gruppe sein, dann wäre das für mich nicht okay.

beide fallen sich ins Wort (Teile unverständlich) Aaron: (lacht) Naja, ihr seht das Problem, oder? (alle lachen zusammen)

Aaron: Ich finde, sie könnten auch ein wenig weniger religiös tun, die nicht Religiösen ein wenig mehr mitmachen, das könnte schon klappen.

Yael: Durchaus. Hm, und man sagt auch so: Zwei Juden, drei Meinungen. (lacht) Man diskutiert da gerne und viel über alles Mögliche. Wie diese Diskussion, die könnten wir sicher noch eine Stunde weiterziehen.

Das kurze „Streitgespräch“ zwischen Yael und Aaron - das zeigte sich in einem Nachgespräch mit den Studierenden - war sehr aufschlussreich, weil es für die Studierenden die verschiedenen Meinungslager und Untergruppen innerhalb der jüdischen Gemeinde sehr gut veranschaulichte. Die eher abstrakte Gliederung der religiösen Strömungen im Judentum, wie wir sie im Seminar behandelt haben (siehe 
Ausführungen dazu weiter oben), wurde hierdurch sehr lebendig und ließ auch Parallelen zur eigenen Erfahrungswelt (z. B. jugendliche Subgruppen, Pfadi-Gruppen) zu.

Für den Verein „Likrat“ führen Yael und Aaron regelmäßig Schulbesuche in Zürich und in den angrenzenden Kantonen durch. Dabei achtet der Verein sehr darauf, dass möglichst verschiedene Jugendliche einen gemeinsamen Schulbesuch durchführen, um die große Spannbreite an religiöser Überzeugung und jüdischem Alltagsleben für Kinder und Jugendliche unmittelbar erfahrbar zu machen. Dass Aaron und Yael sich in ihrer Haltung zum jüdischen Glauben deutlich unterscheiden, obwohl sich beide als "orthodox" bezeichnen, haben die Ausschnitte aus der Diskussion sehr schön veranschaulicht. Vor dem Hintergrund des öffentlichen Engagements der beiden Jugendlichen, stellten wir auch folgende Frage:

Itta: Seht ihr euch in eurer Rolle als LIKRATIJugendliche als jüdische Aktivistinnen?

Yael: Nein.

Aaron: Also, so habe ich noch nie über mich nachgedacht. Nein.

Itta: Nein? Interessant. Ich habe mir gedacht, ihr setzt euch für die Religion, für Öffentlichkeitsarbeit ein.

Yael: $\mathrm{Na}$, es ist vielmehr gegen Antisemitismus.

Itta: Hm. (zustimmend) Sich zu engagieren?

Aaron: Es ist mehr so ein bisschen aufklären. Also, habt ihr den Film, Wolkenbruch" gesehen? (alle zustimmen ja, hm)

Aaron: Ja, also ich habe den auch gesehen und ich habe mich krummgelacht. Es ist wirklich lustig gewesen. Also das jüdische Leben. Ja, ein bisschen schon, also wie sie es zeigen und wie das jüdische Leben ist und die Vorurteile abbauen, die man hat. Der Film vermittelt einen ersten Einblick in das jüdische Leben und das möchte ich ja auch mit Likrat machen.

Auch wenn Yael und Aaron sich vom Begriff „Aktivisimus", wie sie in meiner Frage mitschwingen, distanzieren, so ist ihr persönliches Engagement für einen offenen Dialog unter Jugendlichen in Form von Schulbesuchen ein wertvoller Schritt, um antisemitischem Denken und anti-jüdischen Vorbehalten mit einem niederschwelligen Angebot aktiv entgegen zu wirken.

\section{Fazit}

Ausgehend von dem Buch „Desintegriert euch!“ und seinen provozierenden Thesen versuchte der Beitrag verschiedene Aspekte des „Schweizer Gedächtnistheaters" auszuleuchten. Die Frage danach, wie es um das „Gedächtnistheater" in der Schweiz bestellt ist, kann in diesem Rahmen nur ansatzweise beantwortet werden. Es ist jedoch deutlich geworden, dass ein "Gedächtnistheater" wie es Max Czollek für Deutschland beschreibt, in der Schweiz nicht existiert. Auch die Schweizer Gesellschaft hat sich erst sehr spät mit ihrer eigenen Rolle im Zweiten Weltkrieg auseinandergesetzt. Hierbei wurde u. a. aufgearbeitet welche Verantwortung die Schweiz für die Aufnahme bzw. Ablehnung von Flüchtlingen spielte und wie Schweizer Banken mit den nachrichtenlosen Vermögen von jüdischen Holocaust-Opfern in der Nachkriegszeit umgingen (z. B. Bergier et al. 2002; Maissen 2005). Als nachhaltigen Erfolg dieser nicht ganz einfachen Auseinandersetzung kann man es bezeichnen, dass im aktuellen Lehrplan 21 die Geschichte und die Rolle der Schweiz im Zweiten Weltkrieg differenziert verankert ist und auch Jüdinnen und Juden als religiöse Minderheit als Thema aufgenommen sind. Gerade in großen Städten der Schweiz, wie z. B. Basel oder Zürich, in denen jüdische Gemeinden das Alltagsbild der Städte mitgestalten, können Themen wie Migration, Integration und Segregation sowie das Verhältnis von Minderheiten und Mehrheitsgesellschaft sehr anschaulich mit einem Geschichts- und Geographiebezug unterrichtet werden.

Die ausgewählten Szenen aus unserem Gespräch mit zwei jüdischen Jugendlichen, die für „Likrat" regelmäßig Schulbesuche durchführen, eröffneten uns zudem neue Einsichten. Aaron und Yael vertreten zwei unterschiedliche religiöse Standpunkte, was einen sehr guten Einblick in die heterogene jüdische Gesellschaft gibt. Sie ergänzen sich aber sehr gut, wenn es darum geht, einen Eindruck vom jüdischen Leben als Jugendliche/r zu erhalten. Auch Fragen zu ihrer eigenen Position als jüdische/r Jugendliche/r in unterschiedlichen Gemeinschaften, die sich für den Dialog zwischen Jüdinnen/Juden und Nicht-Jüdinnen/ Juden einsetzen, beantworteten sie uns ausführlich. Diese Einsichten und Erfahrungen schätzten die Studierenden als extrem wertvoll und besonders eindrücklich ein. Als angehende Lehrpersonen für Geographie haben sie im Seminar eine sehr offene Form der Begegnung und des Austausches von unterschiedlichen Jugendlichen kennengelernt. Sowohl inhaltlich als auch didaktisch schätzten die Studierenden ihren Lernerfolg im Seminar als sehr groß ein, gerade weil sie sich mit einem bislang unbekannten Thema auseinandersetzen und andere Formen des Wissenserwerbs und Austausches erfahren konnten.

Dass diese Form des Dialogs für Klassen ab der Sekundarstufe I von „Likrat“ angeboten und organisiert wird, ist sicher eine wertvolle Anregung für Geographie-Lehrpersonen, die ihren Unterricht für solche besonderen Begegnungen öffnen möchten. 


\section{Literatur}

Bergier, J.-F., W. Bartoszewski, S. Friedländer et al. (2002): Die Schweiz, der Nationalsozialismus und der Zweite Weltkrieg. Schlussbericht der Unabhängigen Expertenkommission Schweiz - Zweiter Weltkrieg. Pendo, Zürich.

Bodemann, Y. M. (1996): Gedächtnistheater. Die jüdische Gemeinschaft und ihre deutsche Erfindung. Rothbuch, Hamburg.

Brunschwig, A., R. Heinrichs, K. Huser et al. (2005): Geschichte der Juden im Kanton Zürich: von den Anfängen bis in die heutige Zeit. Orell Füssli, Zürich.

Bundesamt für Statistik (2019): Religionszugehörigkeit, 2015-2017. https://www.bfs.admin.ch/bfs/de/home/ statistiken/bevoelkerung/sprachen-religionen/religionen. html (20.12.2019)

Czollek, M. (2018a): Keine Juden mehr für Deutsche? In: Homolka, W., J. Fegert \& J. Frank (Hrsg.): „Weil ich hier leben will ..." Jüdische Stimmen zur Zukunft Deutschlands und Europas. Herder, Freiburg u.a. S. 84-99.

Czollek, M. (2018b): Desintegriert euch! Carl Hanser Verlag, München.

Fahlbusch, M., M. Rössler \& D. Siegrist (Hrsg.) (1989): Geographie und Nationalsozialismus. 3 Fallstudien zur Institution Geographie im Deutschen Reich und der Schweiz. (=urbs et regio 51). Ghk.

Dreyfuss-Kahn, M. (2013): Abriss der Geschichte der Juden in der Schweiz vom Mittelalter bis heute. In: Familienforschung Schweiz: Jahrbuch 40. S. 79-89.

Gerson, D., S. Bossert, M. Dreyfus et al. (2010): Schweizer Judentum im Wandel (Schlussbericht, NFP 58). Basel.

Guggenheim-Grünberg, F. (1967): Judenschicksale und "Judenschuol“ im mittelalterlichen Zürich. Beiträge zur Geschichte und Volkskunde der Juden in der Schweiz. H. 12. Verlag Jüdische Buch-Gemeinde, Zürich.

Girardet, G. (2001): Der Judenmord von 1349. https:// www.nzz.ch/article77WFU-1.471520 (20.12.2019)

Heske, H. (2008): „... und morgen die ganze Welt“: Erdkundeunterricht im Nationalsozialismus. BoD, Norderstedt.

Hintermann, C. (2019): Who has the right to be remembered? Erinnerungs- und Gedächtnisorte der Migration in Wien. In: Geographische Zeitschrift 107(1). S. 13-36.

Hintermann, C. \& D. Rupnow (2016): Orte, Räume und das Gedächtnis der Migration. Erinnern in der (post-) migrantischen Gesellschaft. Mitteilungen der Österreichischen Geographischen Gesellschaft 158. S. 59-83.

Historisches Lexikon der Schweiz (o. J.): Judentum. http:// www.hls-dhs-dss.ch/textes/d/D11376.php (20.12.2019)

Longchamp, C., J. Dumont, P. Leuenberger et al. (2000): Einstellungen der SchweizerInnen gegenüber Jüdinnen und Juden und dem Holocaust. Eine Studie des GfS-
Forschungsinstituts im Auftrag der Coordination intercommunautaire contre l'antisemitisme et la diffamation (CICAD) und des American Jewish Committee (AJC). Bern.

Maissen, T. (2005): Verweigerte Erinnerung. Nachrichtenlose Vermögen und Schweizer Weltkriegsdebatte 1989-2004. Verlag Neue Zürcher Zeitung, Zürich.

Michel, B. (2014): Antisemitismus, Großstadtfeindlichkeit und reaktionäre Kapitalismuskritik in der deutschsprachigen Geographie vor 1945. In: Geographica Helvetica 69(1). S. 193-202.

Rössler, M. (1986): „Blut und Boden - Volk und Raum“ - Thesen zur Geographie im Nationalsozialismus. In: Friedrichs, J. (Hg.): 23. Deutscher Soziologentag. Westdeutscher Verlag, Opladen. S. 741-744.

Ruoff, U., A. Siegfried, R. Frei-Stolba et al. (1995): Geschichte des Kantons Zürich. Bd. 1. Frühzeit bis Spätmittelalter. Werd Verlag, Zürich.

Schultz, H.-D. (1989): Versuch einer Historisierung der Geographie des Dritten Reiches am Beispiel des geographischen Großstadtdenkens. In: Fahlbusch, M., M. Rössler \& D. Siegrist (Hrsg.): Geographie und Nationalsozialismus. 3 Fallstudien zur Institution Geographie im Deutschen Reich und der Schweiz. (=urbs et regio 51). Ghk. S. 1-75.

Schultz, H.-D. (2017): „Steißpauker“, „Lügen“ und „wehrlose Kinder“: Wie Schulgeographen dazu beitrugen, nach dem Ersten Weltkrieg den Frieden zu verlieren. In: GWUnterricht 148(4). S. 43-57.

Siegrist, D. (1989): Heimat - Landschaft - Nation. Ein Beitrag zur Geschichte der Schweizer Geographie während des deutschen Faschismus. In: Fahlbusch, M., M. Rössler. \& D. Siegrist (Hrsg.): Geographie und Nationalsozialismus. 3 Fallstudien zur Institution Geographie im Deutschen Reich und der Schweiz. (=urbs et regio 51). Ghk. S. 275-423.

Statista (2019): Anzahl der Juden in der Schweiz nach Kantonen im Jahr 2017. https://de.statista.com/statistik/ daten/studie/322654/umfrage/anzahl-der-juden-in-derschweiz-nach-kantonen (20.12.2019)

Stadt Zürich Statistik (2019): Analyse Stadtzürcher Religionslandschaft 2019. https://www.stadt-zuerich.ch/prd/ de/index/statistik/publikationen-angebote/publikationen/Analysen/A_001_2019.html (20.12.2019)

Statistik Stadt Zürich (2019): Bevölkerung. https://www. stadt-zuerich.ch/prd/de/index/statistik/themen/bevoelkerung.html (20.12.2019)

Weill, R. (2004): Strukturelle Veränderungen der Schweizer Judenheit. In: Jüdische Lebenswelt Schweiz, 100 Jahre Schweizerischer Israelitischer Gemeindebund. S. 120-129.

Wurz, J. (2013): Rückblick auf die Kontroverse um die Holocaust-Gelder. https://www.swissinfo.ch/ger/politik/ zwei-revisionen-_rueckblick-auf-die-kontroverse-umdie-holocaust-gelder/36759886 (13.9.2019) 